\title{
Saving Limbs in Diabetics: Challenges and Opportunities
}

\author{
Zia Ur Rehman \\ Department of Surgery, The Aga Khan University Hospital, Karachi, Pakistan
}

Diabetic patients have 15 to 30 times higher risk of amputation compared to non-diabetics. ${ }^{1}$ About $85 \%$ of diabetic patients with a non-healing foot ulcer end up in major amputations. ${ }^{2}$ Diabetic foot ulcers (DFU) are due to combination of neuropathy, peripheral vascular disease (PAD) and infection. ${ }^{3}$ Detection of PAD in an ulcerated foot is paramount as it helps in early revascularisation. The classic presentations of chronic limb ischemia, such as rest pain and intermittent claudication, are masked due to associated neuropathy in these patients; and the very first presentation may be with a gangrenous toe or a non-healing ulcer. Ischemia remains untreated in these patients before significant time has lapsed. Early detection and timely referral to dedicated centres with vascular surgery services is the key in the management of these patients. What might have been sufficient circulation for an intact, uninfected foot may be inadequate once ulceration, sepsis and gangrene develop. Ulcer formation in an ischemic leg, is a sign of severe chronic limb ischemia; and leaving it untreated, results in major limbloss.

Macroangiopathy in diabetics typically involves distal superficial femoral artery and infra-popliteal arteries, which is usually severe, diffuse and multi-segmental. Most patients are candidates for surgical or endovascular revascularisation.

Infra-inguinal bypasses, using veins, have excellent limb salvage rate with minimal perioperative morbidity and mortality. These are reserved for patients with good life expectancy and who are fit for anesthesia. Inline flow to the ankle or foot via at least one of the pedal arteries is required for wound healing. An adequate size ( $>3 \mathrm{~mm}$ ), and good quality (without fibrosis and stenosis) vein is pre-requisite for these procedures which can be mapped preoperatively. Vein can be used in reversed, non-reversed or in situ fashion. Bypasses to foot arteries also produce excellent long-term patency and good limb salvage rate. ${ }^{4}$

Endovascular therapy has evolved as an alternative with encouraging results; even for patients who are not fit for surgery.

Correspondence to: Dr. Zia Ur Rehman, Department of Surgery, The Aga Khan University Hospital, Stadium Road, Karachi, Pakistan

E-mail: ziaur.rehman@aku.edu

Received: January 21, 2020; Revised: September 14, 2020;

Accepted: September 14, 2020

DOI: https://doi.org/10.29271/jcpsp.2020.10.1003
The clinical outcomes of wound healing and amputation are comparable with open interventions. Endovascular techniques, such as percutaneous transluminal angioplasty (PTA) with and without stenting, subintimal angioplasty, drug coated balloons and stents and atherectomy devices have established their roles with good results. Drug-eluted balloons and stents prevent neointimal hyperplasia and re-stenosis. Sirolimus-eluting stents have shown to inhibit neointimal hyperplasia in the coronary vasculature. Atherectomy devices debulk heavy and calcified lesions to facilitate routine angioplasty in difficult lesion.

The lesion-crossing techniques have also improved. Antegrade true lumen crossing has superior patency rate. ${ }^{5}$ Subintimal approach, retrograde access via pedal arteries and subintimal arterial flossing with antegrade-retrograde intervention (SAF$A R I)$ are some of the innovative techniques in crossing lesions which are not crossable by routine 'antegrade' approach. ${ }^{6}$ These all help in improving the primary patency and limb salvageability.

Limb salvage rate of $80-85 \%$ has been reported using either surgical, endovascular or hybrid (combination of two) revascularisation techniques in these patients. ${ }^{7}$ Fast-track programme consisting of early endovascular revascularisation and local surgical treatment have been reported to reduce amputations in DFU patients. ${ }^{8}$

"Time is tissue," and diabetic foot problems requiring a timely, team-approach to prevent amputation. Organised programmes have shown reduced amputations in developed world. ${ }^{9}$ The same model customised with local needs can be incorporated in lowto-middle-income countries to produce these results.

Limb salvage is a philosophy with a mindset to assess every diabetic foot patient for compromised circulation and possible revascularisation before offering minor or major amputation, unless there is spreading infection. Ischemia should be managed to prevent further soft tissue loss and improve the likelihood of wound healing. After ischemia and infection are managed, the pressure needs to be relieved from the foot to prevent recurrent ulcer formation in these patients. There is need to generate this awareness among medical community, especially the surgeons managing these patients. It will definitely improve the outcomes of these patients.

\section{REFERENCES}

1. Lin JH, Jeon SY, Romano PS, Humphries MD. Rates and timing of subsequent amputation after initial minor 
amputation. J Vasc Surg 2020; 72(1):268-75. doi: 10.1016/ j.jvs.2019.10.063.

2. Tan WS, Arulselvan P, Ng SF, Mat Taib CN, Sarian MN, Fakurazi S. Improvement of diabetic wound healing by topical application of Vicenin-2 hydrocolloid film on Sprague Dawley rats. BMC Complement Altern Med 2019; 19(1):20. doi: 10.1186/s12906-018-2427-y.

3. Lavery LA, Oz OK, Bhavan K, Wukich DK. Diabetic Foot Syndrome in the Twenty-First Century. Clin Podiatr Med Surg 2019; 36(3):355-9. doi: 10.1016/j.cpm.2019.02.002.

4. Saarinen $E$, Kauhanen $P$, Söderström M, Albäck A, Venermo M. Long-term Results of Inframalleolar Bypass for Critical Limb Ischaemia. Eur J Vasc Endovasc Surg 2016; 52(6): 815-22. doi: 10.1016/j.ejvs.2016.08.051.

5. Choke E, Tang TY, Cheng SC, Tay JS. Treatment of lower limb ischaemia in patients with diabetes. Diabetes Metab Res Rev 2020; 36 Suppl 1:e3262. doi: 10.1002/dmrr.3262.
6. Dinoto E, Pecoraro F, Mirabella D, Ferlito F, Farina A, Biundo $\mathrm{NL}$, et al. A single-center experience on below-the-knee endovascular treatment in diabetic patients. Transl Med UniSa 2020; 21:21-3.

7. Hinchliffe RJ, Brownrigg JR, Andros G, Apelqvist J, Boyko EJ, Fitridge $R$, et al. Effectiveness of revascularization of the ulcerated foot in patients with diabetes and peripheral artery disease: A systematic review. Diabetes Metab Res Rev 2016; 32 Suppl 1:136-44. doi: 10.1002/dmrr.2705.

8. Troisi N, Ercolini L, Chisci E, Frosini P, Pigozzi C, Enrico B, et al. Use of tapered balloons to recanalize occluded belowthe-knee arteries in diabetic patients with critical limb ischemia. Ann Vasc Surg 2016; 31:105-10. doi: 10.1016/ j.avsg.2015.08.015.

9. Rogers LC, Bevilacqua NJ. Organized programs to prevent lower-extremity amputations. J Am Podiatr Med Assoc 2010; 100(2):101-04. doi: 10.7547/1000101. 